\title{
Video-assisted thoracoscopic surgery for large anterior mediastinal hematoma
}

\author{
Yuhang Ruan, Xiaodong Li, Jianfei Shen, Minhua Ye \\ Department of Thoracic Surgery, Taizhou Hospital of Zhejiang Province, Wenzhou Medical University, Linhai 317000, China \\ Correspondence to: Minhua Ye; Jianfei Shen. Department of Thoracic Surgery, Taizhou Hospital of Zhejiang Province, Wenzhou Medical University, \\ No. 150 Ximen Street, Linhai 317000, China. Email: ymh@enzemed.com; jianfei051@163.com.
}

Submitted Sep 03, 2019. Accepted for publication Dec 10, 2019.

doi: $10.21037 /$ jtd.2019.12.95

View this article at: http://dx.doi.org/10.21037/jtd.2019.12.95

Anterior mediastinal bleeding caused by blunt chest injury is dangerous. Patients with rupture of large cardiac vessels often die of massive blood loss before getting to a hospital. The internal thoracic artery and vein, intercostal vessels or fracture ends caused bleeding that resulted in a mediastinal hematoma $(1,2)$. Due to the nature of blunt chest injuries, the variation of anterior mediastinal hematoma cases is wide, and the specific source of bleeding is not always clear. The most commonly utilized methods of treatment are thoracotomy and hemostasis, and vascular interventional embolism (3). Video-assisted thoracoscopic surgery (VATS) is rarely reported as being used in the treatment of acute traumatic anterior mediastinal hematoma. This paper reports a case of traumatic giant anterior mediastinal hematoma treated by thoracoscopy.

The case was a 51-year-old male patient who accidentally fell 13 hours before admission. Emergency chest CT showed an anterior mediastinal hematoma, pericardial and bilateral pleural effusions (Figure 1). On cardiac color Doppler ultrasound, no obvious traumatic abnormalities were found in the large blood vessels or cardiac structure. Body examination: left edge tenderness of the upper sternal segment was evident. Sounds on percussion were turbid. There were no obvious bone scraping sounds on the chest wall. Heart rate was 113 beats $/ \mathrm{min}$ and blood pressure was $85 / 66 \mathrm{mmHg}(1 \mathrm{mmHg}=0.133 \mathrm{kPa})$. Considering the patient's condition, the decision was made to perform emergency surgery.

After general anesthesia, the right trunk was elevated, so that the patient tilted to the left $30^{\circ}-45^{\circ}$. A $1.5 \mathrm{~cm}$ incision was made in the fifth intercostal space at the right midaxillary line to insert an endoscopic lens. About 0.5 and $1.5 \mathrm{~cm}$ incisions were made in the third intercostal space of the right anterior axillary line and the fifth intercostal space at the midclavicular line for access to the surgical site. The findings were as follows: right thoracic hemorrhage, massive anterior mediastinal hematomas (extending from the superior vena cava to the diaphragm), and a large amount of blood clots within the mediastinal pleura. The hematomas were completely removed along with the thymus and anterior mediastinal fat. Active hemorrhage of posterior sternal arterioles was seen. A titanium clip, prolene thread sutures, electrocoagulation and an ultrasound knife were used to stop the bleeding completely, and the left pleura was opened across the mediastinum to remove left pleural hemorrhage (Figures 2,3). At the end of the operation, the closed thoracic drainage tube was retained on the right side and the thoracic drainage tube on the left side was kept in place after the body position was reversed.

Chest CT showed good recovery both 3 days after the operation and 6 days after the operation. The patient discharged from hospital on the 7 th day following the operation. The patient returned 6 weeks after the operation for a follow-up CT; the patient recovered well and did not have any complaints.

\section{Discussion}

Why was the right chest approach chosen for this thoracoscopic surgery? (I) Preoperative examination basically excluded injury of large cardiac vessels and severe rib fracture, and intraoperative bleeding was expected to be controlled. (II) Patients with massive anterior mediastinal hematomas often undergo median sternal thoracotomy, which may lead to hematoma rupture during sternotomy. (III) Thoracoscopy enters the chest from the minor injury 

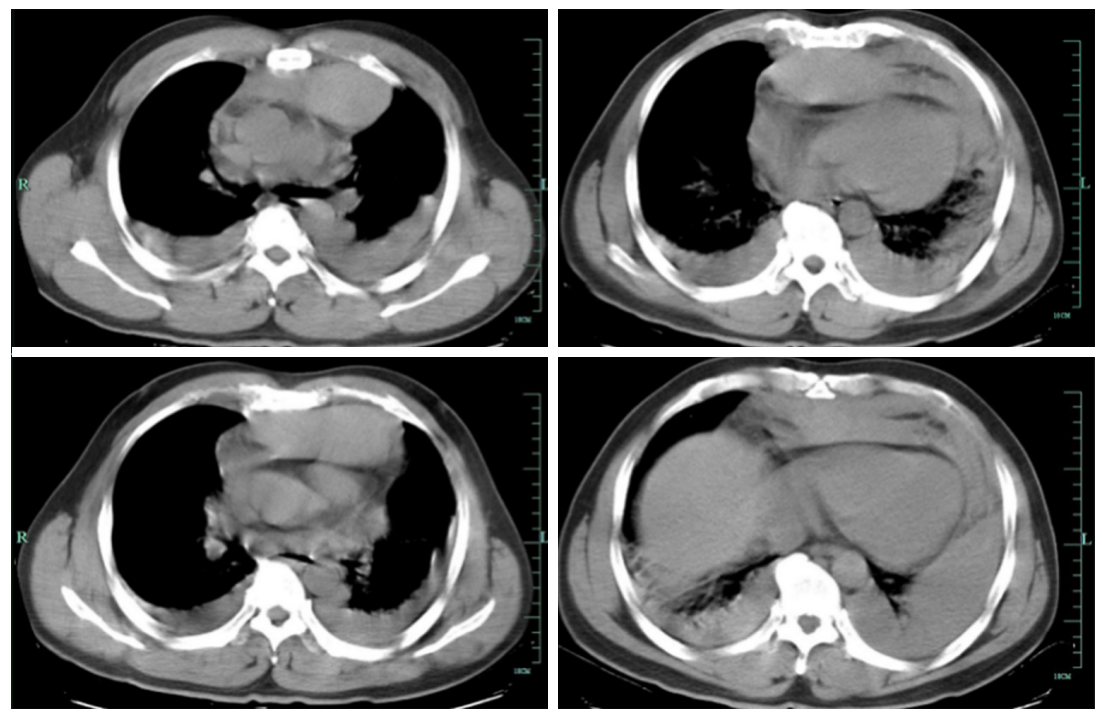

Figure 1 Preoperative chest CT indicates mediastinal hematoma, peridial and bilateral pleural effusions, right first costal cartilage fracture. There were no abnormalities seen on CT scan of the upper abdomen.
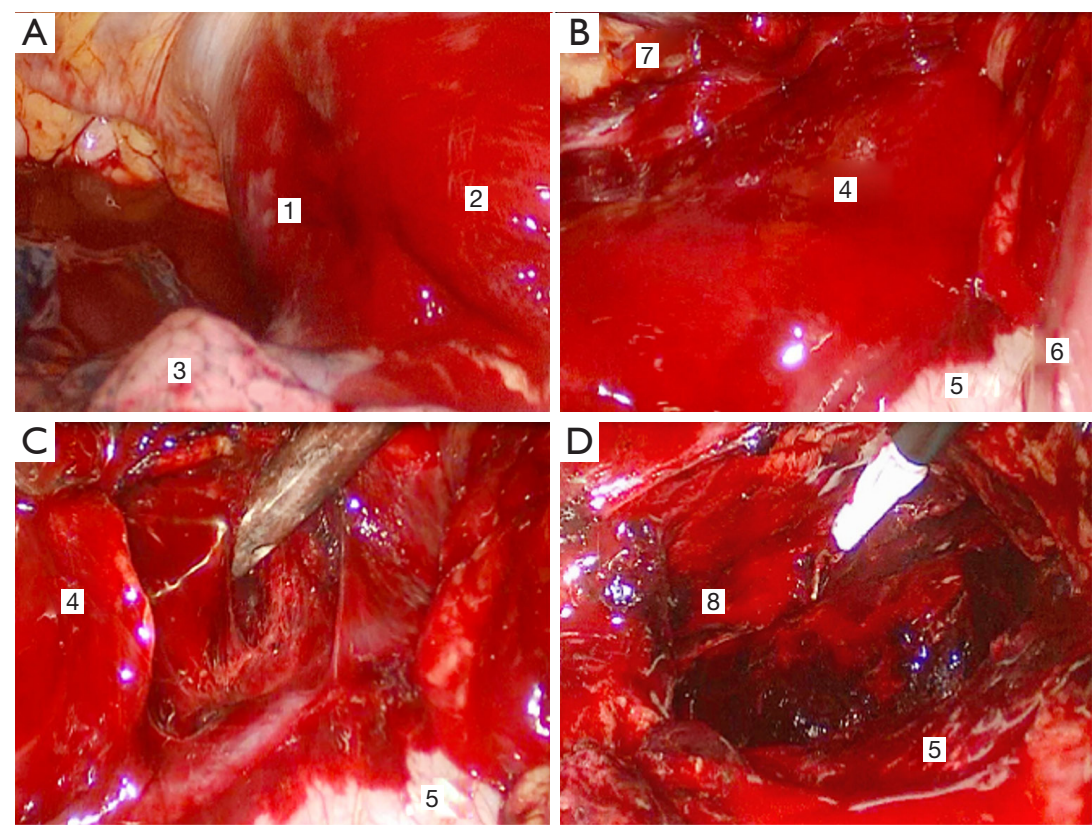

Figure 2 Anterior superior mediastinal hematomas (A), anterior inferior mediastinal hematomas (B), opened mediastinal hematomas with blood clots and loose anterior mediastinal tissue (C), hemostasis of active bleeding point behind sternum (D). Notes: 1, superior vena cava; 2, hematomas superior to anterior mediastinum; 3, left upper lung; 4, hematomas inferior to anterior mediastinum; 5, pericardium; 6, Diaphragm; 7, anterior chest wall; 8, point of active bleeding posterior to sternum 

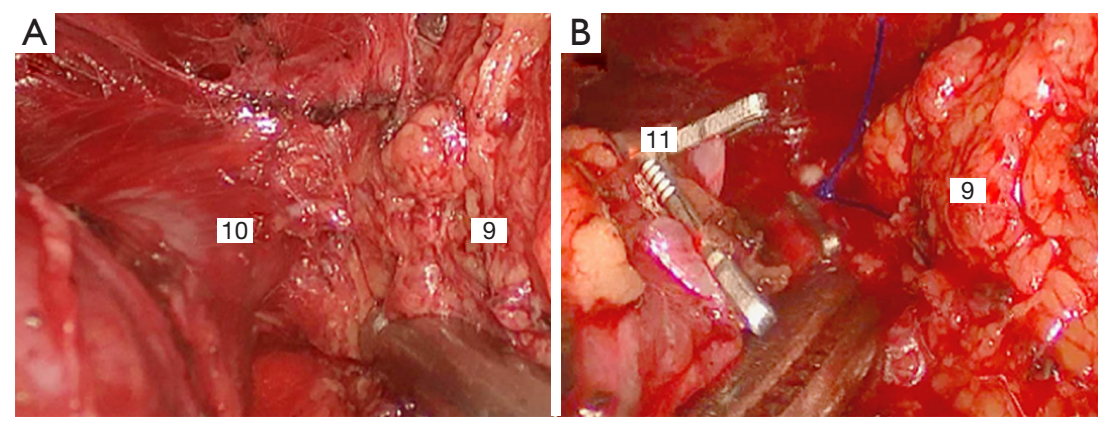

Figure 3 Minor vein and thymic tissue (A), hemostasis of pericardial surface beside nameless vein with titanium clip and suture (B). Notes: 9, thymus and anterior mediastinal fat; 10, minor vein; 11, titanium clamp and suture for hemostasis.

side of the chest wall to avoid the area of trauma and minimize the aggravation of bleeding. (IV) The median sternal incision should be sterilized thoroughly before the operation. If there is uncontrollable bleeding in the anterior mediastinum or left chest during the operation, the patient can be quickly converted to the supine position or the right tilting position. Thoracoscopy has many advantages, however, because of its moderate inconvenience in emergency situations with massive bleeding or requiring fine suturing, it is not often used in the treatment of acute traumatic mediastinal hematomas $(4,5)$.

The thoracoscopic approach should be considered as an alternative surgical option, provided sufficient preoperative evaluation is done and cardiac macrovascular injury is excluded, and emergency thoracotomy is prepared for as a backup option.

\section{Acknowledgments}

Thanks Kaya Petersen for his assistance in language retouching.

Funding: None.

\section{Footnote}

Conflicts of Interest: The authors have no conflicts of interest to declare.

Ethical Statement: The authors are accountable for all aspects of the work in ensuring that questions related to the accuracy or integrity of any part of the work are appropriately investigated and resolved. Written informed consent was obtained from the patient for publication of this manuscript and any accompanying images.
Open Access Statement: This is an Open Access article distributed in accordance with the Creative Commons Attribution-NonCommercial-NoDerivs 4.0 International License (CC BY-NC-ND 4.0), which permits the noncommercial replication and distribution of the article with the strict proviso that no changes or edits are made and the original work is properly cited (including links to both the formal publication through the relevant DOI and the license). See: https://creativecommons.org/licenses/by-nc-nd/4.0/.

\section{References}

1. Yeh DD, Hwabejire JO, DeMoya MA, et al. Sternal fracture--an analysis of the National Trauma Data Bank. J Surg Res 2014;186:39-43.

2. Odell DD, Lipsky AM, Sidharta P. Sternal fracture, a sentinel finding in trauma: An analysis of 1863 cases. Journal of American College of Surgeons 2011;213:S39.

3. Albors J, Estigarribia AJ, Llorens R. Delayed pseudoaneurysm of the internal mammary artery: conservative management. Neth Heart J 2012;20:428-9.

4. Mizumoto M, Oizumi H, Kato H, et al. Thoracoscopic surgery for traumatic mediastinal hematoma. Kyobu Geka 2013;66:374-8.

5. Samiatina D, Rubikas R. Video-assisted thoracoscopic surgery as an alternative to urgent thoracotomy following open chest trauma in selected cases. Medicina (Kaunas) 2004;40 Suppl 1:134-8.

Cite this article as: Ruan Y, Li X, Shen J, Ye M. Video-assisted thoracoscopic surgery for large anterior mediastinal hematoma. J Thorac Dis 2020;12(3):1133-1135. doi: 10.21037/jtd.2019.12.95 